TITLE:

\title{
Dynamics of Proton Transfer and Vibrational Relaxation in Dilute Hydrofluoric Acid
}

$\operatorname{AUTHOR}(\mathrm{S})$ :

Joutsuka, Tatsuya; Ando, Koji

\section{CITATION:}

Joutsuka, Tatsuya ...[et al]. Dynamics of Proton Transfer and Vibrational Relaxation in Dilute Hydrofluoric Acid. The Journal of Physical Chemistry A 2011, 115(5): 678-684

ISSUE DATE:

2011-01-06

URL:

http://hdl.handle.net/2433/217073

\section{RIGHT:}

This document is the Accepted Manuscript version of a Published Work that appeared in final form in 'The Journal of Physical Chemistry A', copyright @ $\odot$ American Chemical Society after peer review and technical editing by the publisher. To access the final edited and published work see http://dx.doi.org/10.1021/jp108413p.; この論文は出版社版でありま せん。引用の際には出版社版をご確認ざ利用ください。; This is not the published version. Please cite only the published version. 


\title{
Dynamics of Proton Transfer and Vibrational Relaxation in Dilute Hydrofluoric Acid
}

\author{
Tatsuya Joutsuka and Koji Ando \\ Department of Chemistry, Kyoto University, Sakyo-ku, Kyoto 606-8502, Japan
}

\begin{abstract}
The molecular mechanisms in both vibrational relaxation and proton transfer (PT) associated with infrared (IR)-induced PT in a dilute hydrofluoric acid solution at ambient temperature are studied by molecular dynamics (MD) simulations with the multistate empirical valence bond model. To investigate the solvation dynamics, a collective solvent coordinate and its perpendicular bath modes are defined from the diabatic energy gap and their motions are examined by the generalized Langevin equation (GLE) formalism. The GLE analysis using the equilibrium MD simulation shows that the major solvent reorganizations in the PT are represented by the water libration and hindered translation. In particular, the water libration gives the stronger coupling to the solvent reorganization and the faster relaxation. The nonequilibrium MD simulation demonstrated that both the HF stretching vibration and the solvent reorganization relax on a similar time scale and thus compete in the PT. It also supported the "presolvation mechanism" for the PT in this system.
\end{abstract}

\section{Introduction}

Proton transfer and vibrational relaxation in water are of fundamental importance in chemistry and biology. Our understanding of these phenomena has been enriched by recent spectroscopic and theoretical studies on ultrafast phenomena. Among them, the possibility of IR-induced $\mathrm{HF}$ ionization ${ }^{1-4}$ is intriguing in the light of acid-base chemistry as well as application of the ground-state coherent control in condensed phases. ${ }^{5,6}$ As an illustration, we show Figure 1 displaying the free energies of the proton transfer $(\mathrm{PT})$ reaction

$$
\mathrm{FH} \cdots \mathrm{H}_{2} \mathrm{O}(1: \text { Reactant }) \rightleftharpoons \mathrm{F}^{-} \cdots \mathrm{H}_{3} \mathrm{O}^{+}(2 \text { : Product })
$$

including the ground $v=0$ and first excited $v=1$ proton vibrational levels, labeled as $\mathrm{G}_{0}$ and $\mathrm{G}_{1}$, versus the solvent coordinate. ${ }^{2,4}$ It shows that the Franck-Condon vibrational excitation $v=0 \rightarrow 1$ of the undissociated HF places the system at an unstable point on the upper curve $\mathrm{G}_{1}$. The solvent will then relax to the bottom of this curve. An ensuing nonadiabatic deexcitation to the ground state surface $G_{0}$ places the system in the transition state (TS) region on the $G_{0}$ surface. From there, the solvent motion can produce the product contact ion pair (as well as the original reactant pair), and thereby enhance the PT rate. Experimentally, this will be achieved by exciting the stretching mode of HF by an IR pulse in the electronic ground state, which provides a fascinating possibility for vibrational photochemistry in solution.

Accordingly, in this work, we analyze the dynamics of vibrational relaxation and PT in a dilute hydrofluoric acid solution with emphasis on the IR-induced PT. Our goal is to provide a 


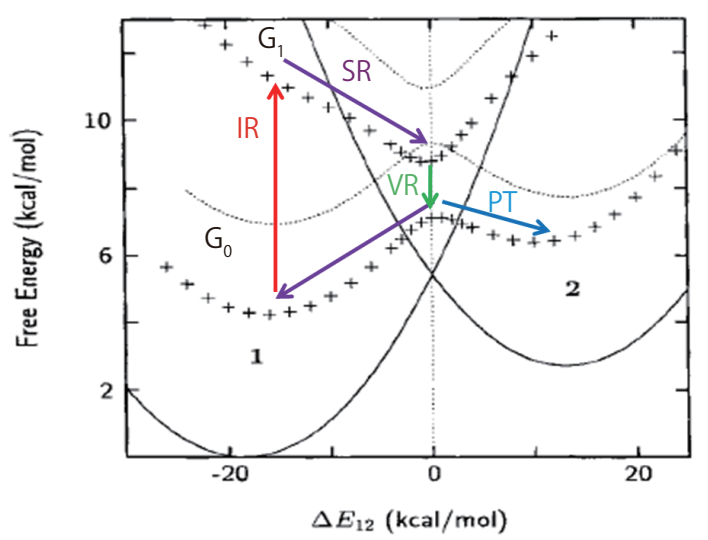

Figure 1: Diabatic free energy curves in the solvent (energy gap) coordinate $\Delta E_{12}$, with the lowest two proton vibrational levels including the solvent self-free-energy (+++). The texts herein, IR, SR, VR, and PT represent infrared excitation, solvent reorganization, vibrational relaxation, and proton transfer, respectively. The 1 and 2 means the reactant and product (contact ion pair) states. Data was taken from ref 4.

molecular-level description of the competition between vibrational relaxation and solvent reorganization, which control this condensed-phase reaction. To this end, we carry out equilibrium and nonequilibrium molecular dynamics (MD) simulations using the multistate empirical valence bond (MS-EVB) model developed and described in a separate article. ${ }^{7}$

The dynamic solvent effects are analyzed using the equilibrium MD results through the reaction path model that extracts and separates the solvent coordinate and the perpendicular bath modes. Furthermore, to analyze the solvation and vibrational relaxation dynamics, the nonequilibrium MD simulation is conducted and compared with the equilibrium MD results. This is because the IR-induced PT involves a highly anharmonic potential energy surface (PES) unreachable by the reaction path model, especially near the TS. For the IR-induced PT to occur via the mechanism outlined above, the vibrational lifetime should be longer than the solvent reorganization time. Therefore, we analyze the competition between the solvent reorganization and the quenching (relaxation) of the vibrational excitation. Specifically, we follow the solvent reorganization dynamics to observe the relaxation after IR excitation.

The outline of this paper is as follows: In section 2, we analyze the vibrational frequency and perform the reaction path analysis using the generalized Langevin equation (GLE) formalism. In section 3, the competition between of the solvent reorganization and vibrational relaxation dynamics in the IR-induced PT is discussed by the nonequilibrium MD. In section 4, the conclusion is given.

\section{Equilibrium MD Simulation}

The equilibrium MD simulation is carried out in this section to analyze the vibrational frequency of the Fourier-transformed velocity autocorrelation function (ACF; section 2.1) and the reaction path using the GLE formalism (section 2.2). The details of the equilibrium simulation are identical to those in ref 7 in which the development of the MS-EVB model for the present system is also described. In particular, the model has been demonstrated to yield reasonable hydration structures and activation energy for the PT. 

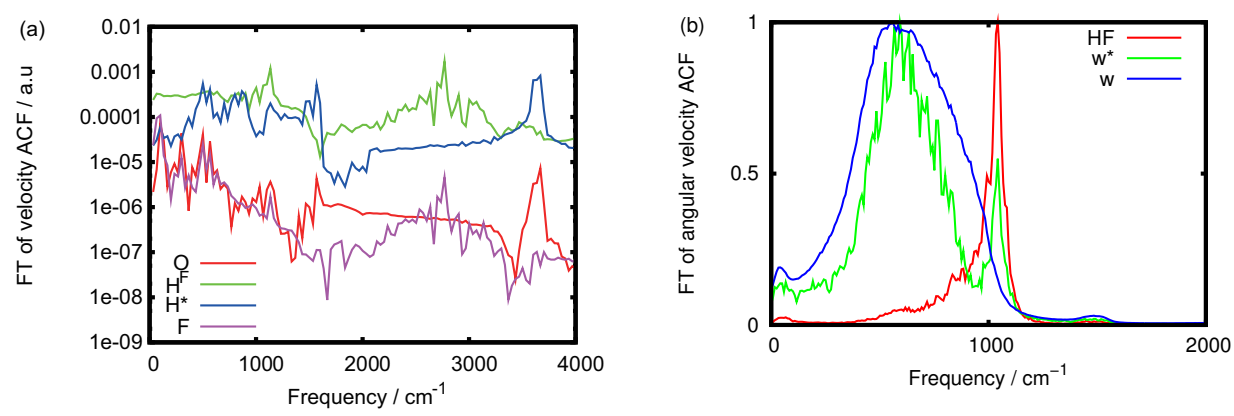

Figure 2: (a) Fourier transform (FT) of the velocity autocorrelation functions (ACFs) of the atoms of HF and its nearest-neighbor water molecule. Note the log-scale of the ordinate. The labels of atoms in the legend are given in Figure 3. (b) Fourier transform of the angular velocity $\mathrm{ACF}$ of HF, $\mathrm{w}^{*}$, and other water molecules (w). Shown is the normalized absolute value of the spectrum.

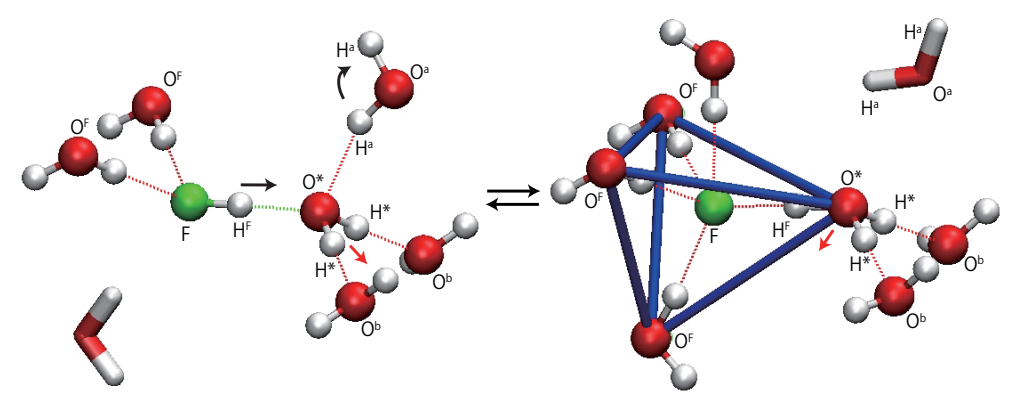

Figure 3: Schematic illustration with labels of HF and water molecules in the vicinity of the proton accepting water and fluorine atom in the course of PT. The red arrow indicates the dipole moment direction of the water or hydronium ion labeled with ${ }^{*}\left(\mathrm{w}^{*}\right)$. The molecules that are not so close to be $\mathrm{H}$-bonded but located in the corresponding directions are drawn by the stick model. These graphics were created using VMD. ${ }^{8}$

\subsection{Vibrational Frequency Analysis}

Before addressing the reaction path analysis, we examine the velocity ACF spectra of the atoms of $\mathrm{HF}$ and its nearest-neighbor water molecule displayed in Figure 2a and b. The configuration and labels of HF and water molecules are shown in Figure 3. The water molecule with the superscript * (hereafter, referred to as $\mathrm{w}^{*}$ ) is that directly H-bonded to the HF molecule. The low signal-to-noise ratio in Figure 2 is due to the use of a logarithmic plot.

The velocity ACF spectra of $\mathrm{H}^{*}$ (blue) and $\mathrm{O}$ (red) in Figure 2a have peaks at 3500-3700 $\mathrm{cm}^{-1}$ corresponding to the $\mathrm{OH}$ stretch. The $\mathrm{H}^{\mathrm{F}}$ (green) and $\mathrm{F}$ (pink) spectra have a relatively broad peak at 2500-3000 $\mathrm{cm}^{-1}$, which can be assigned to the HF stretch. The absence of the $\mathrm{H}^{*}$ and $\mathrm{O}$ peaks in that region is also noted. This HF stretching frequency is close to that found in the previous calculation, ${ }^{4} 2520 \mathrm{~cm}^{-1}$. In the same vein, recent experiments and Car-Parrinello (CP) MD calculations have shown that the HF frequencies at cryogenic temperatures ${ }^{9,10}$ and in dilute HF solution at room temperature (isotopically shifted) ${ }^{11}$ are in the region from 2400 to $2800 \mathrm{~cm}^{-1}$.

The $\mathrm{H}^{\mathrm{F}}$ (green) spectrum in Figure 2a has a peak at $\sim 1100 \mathrm{~cm}^{-1}$ that corresponds to the HF libration. This assignment is confirmed by the ACF spectra of the angular velocity (around 


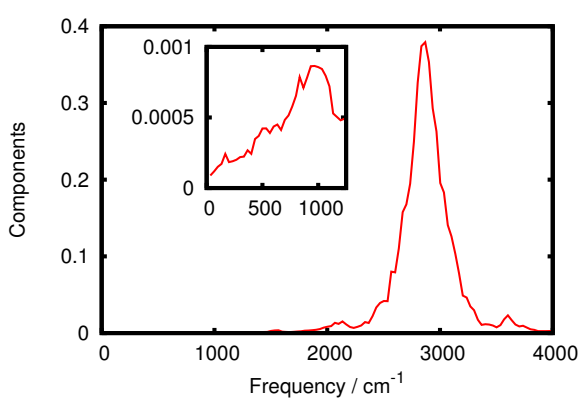

Figure 4: Components of the reaction (solvent) coordinate vector $\tilde{s}_{i}^{2}$, calculated by eq 10 , along the frequency. The inset enlarges the low frequency region.

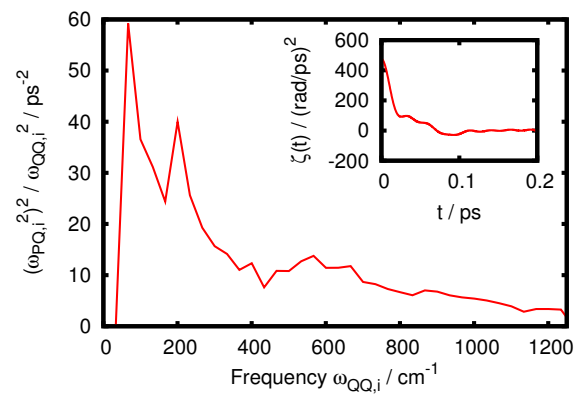

Figure 5: Fourier transformed friction kernel in eq 7, $\left(\omega_{P Q, i}^{2}\right)^{2} / \omega_{Q Q, i}^{2}$ along $\omega_{Q Q, i}$, computed with a cutoff below $1250 \mathrm{~cm}^{-1}$ in Figure 4 . The inset is the time-dependent friction kernel.

the center of mass) of HF, $w^{*}$, and other water molecules, which is shown in Figure $2 \mathrm{~b}$. This librational frequency is also in agreement with the previous MD calculation. ${ }^{12}$ In Figure $2 b$, the water libration can be seen in the broad spectra peaked at 560 and $590 \mathrm{~cm}^{-1}$ for $\mathrm{w}^{*}$ and other water molecules, respectively. The spectrum of $\mathrm{w}^{*}$ has a peak also at $1040 \mathrm{~cm}^{-1}$, which is caused by the strong coupling to HF.

\subsection{Reaction Path Analysis}

Using the reaction path model, ${ }^{13-15}$ we analyze the collective solvent motion and its perpendicular components in the PT. The formulation is summarized in the Appendix. Figure 4 shows the components of reaction (solvent) coordinate vector calculated from eq 10 and its blowup in the low frequency region (inset). In Figure 5, we show the friction kernel (eq 7) of the GLE (eq 6 ) and its Fourier cosine transform.

In this calculation and hereafter, the solvent coordinate is defined by the vertical energy gap of the diabatic solute-solvent interactions between the covalent and ionic valence bond (VB) states. The diabatic solute-solvent interactions mean $U^{\mathrm{H}_{3} \mathrm{O}^{+} / \mathrm{H}_{2} \mathrm{O}}+U^{\mathrm{F}^{-} / \mathrm{H}_{3} \mathrm{O}^{+}}+U^{\mathrm{F}^{-} / \mathrm{H}_{2} \mathrm{O}}$ and $U^{\mathrm{HF} / \mathrm{H}_{2} \mathrm{O}}$ defined by eqs 2 and 6 in ref 8 . In this way, the solvent coordinate depends on the solvent configuration. This definition is similar in idea but different from that used by Ando and Hynes. ${ }^{4}$ It is also different from that used by Staib et al. ${ }^{16}$ in that the product energy is defined by the lowest eigenvalue among ionic VB states (as we employ the multistate EVB). Another difference is that since the Hamiltonian is not averaged by the proton wave function, the solvent coordinate contains the proton coordinate dependence. To eliminate this dependence and to 


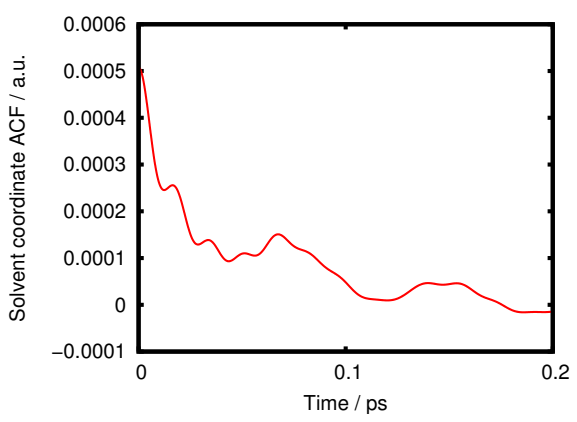

Figure 6: Autocorrelation function (ACF) of the solvent coordinate.

compare with the previous study, we have cut off the high frequency region of the intramolecular vibrations and used only the Fourier components below $1250 \mathrm{~cm}^{-1}$, which is shown in the inset of Figure 4.

The effective mass (eq 5), effective frequency (eq 8), and characteristic frequency (eq 9) of the GLE are calculated as $\mu=2.1 \times 10^{-6} \mathrm{ps}^{2} / \mathrm{eV}, \Omega=540 \mathrm{~cm}^{-1}$, and $\bar{\omega}=905 \mathrm{~cm}^{-1}$. The difference of the effective frequency from those in the previous related works (dielectric relaxation of water associated with ionization of $N, N$-dimethylaniline (DMA) ${ }^{13}$ and photoinduced electron transfer between DMA and anthracene in acetonitrile solution, ${ }^{15}$ which yielded $\Omega=417$ and $92 \mathrm{~cm}^{-1}$, respectively) stems from the vibrational modes involved in the solvent coordinate. Similarly, the discrepancy of the average frequency from the previous ones $(\bar{\omega}=794$ and 219 $\mathrm{cm}^{-1}$ ) arises from the coupling to the bath in addition to the vibrational modes involved. In our simulation, both $\Omega$ and $\bar{\omega}$ are higher because the relatively high frequency modes from the water and HF librations are involved in the solvation process.

The different effective mass (from $\mu=4.2 \times 10^{-5}$ and $9.5 \times 10^{-4} \mathrm{ps}^{2} / \mathrm{eV}$ in the previous works) seems to arise from the different electronic adiabaticity in PT and ET. That is, as seen in eq 5 , the lower $\mu$ means that the coupling of the bath to the solvent coordinate is stronger. The strong coupling in the present system can come from the direct vibrational interactions, ${ }^{16}$ especially in the water and HF librations around $1000-1100 \mathrm{~cm}^{-1} \mathrm{~b}$ as discussed later. In addition, the effective frequency $\Omega$ without cutoff and by the Gaussian approximation $\Omega^{\prime}=\left(\left\langle s^{2}\right\rangle /\left\langle\dot{s}^{2}\right\rangle\right)^{1 / 2}$ are computed to be 2872 and $2743 \mathrm{~cm}^{-1}$. The degree of this accordance between $\Omega$ and $\Omega^{\prime}$ indicates a measure of the adequacy of the harmonic bath model.

The components in Figure 4 are mainly composed of the $\mathrm{HF}$ and $\mathrm{OH}$ stretches, and the water and HF librations. A small flat tail at the low frequency region can also be seen, which would be the contribution from hindered translation $\left(60-200 \mathrm{~cm}^{-1}\right)$. The HF libration (1000$1100 \mathrm{~cm}^{-1}$ ) has the larger intensity than the water libration $\left(500-1000 \mathrm{~cm}^{-1}\right.$; see Figure $2 \mathrm{~b}$ ). On the other hand, Figure 5 shows the larger intensity in the two lowest-frequency components. Therefore, whereas the reaction coordinate is dominated by the HF and water librations in the intermolecular interactions, the bath modes coupled to them are mainly composed of the hindered translation. As seen in the figure, the time-dependent friction kernel shows a rapid decay, descend down to negative value, and accompany a small oscillation.

The autocorrelation function (ACF) of the solvent coordinate is also shown in Figure 6 to investigate the characteristic time scale of the solvent coordinate. The fitting by exponential function gives the solvent reorganization time $\tau_{\mathrm{s}}=58 \mathrm{fs}$. This is faster than the solvent reorganization time scale of water, $\tau_{\mathrm{s}} \sim 100 \mathrm{fs} .{ }^{17}$

Although we have performed here a simple analysis using the time progression of the sol- 
vent coordinate computed from the equilibrium MD simulation, more direct information about the dynamics on the potential surfaces around the crossing seam (TS) can be obtained by the nonequilibrium MD simulation below.

\section{Nonequilibrium MD Simulation}

The nonequilibrium MD trajectory calculation is carried out in this section to investigate the nonequilibrium solvent reorganization (section 3.1) and the vibrational relaxation (section 3.2). The configurations and velocities of the system used hereafter, which are to be adopted as the initial condition for the nonequilibrium trajectory calculations, were sampled in the course of the equilibrium MD simulation on the ground state potential.

Here, we adopt two types of initial conditions to simulate IR excitation for respective purposes. In section 3.1, we increase the velocity of the HF stretching mode in the direction that the HF distance extends by the amount that the kinetic energy increases by $10.1 \mathrm{kcal} / \mathrm{mol}$ corresponding to $2500 \mathrm{~cm}^{-1}$. This initial condition is to cause the PT and solvent reorganization forcibly. The dissociation of HF was observed in $2.3 \%$ of 1000 nonequilibrium MD trajectories, and these reactive trajectories are averaged to discuss the solvent reorganization dynamics. In section 3.2, 1000 quenched (undissociated) trajectories are averaged to discuss the vibrational relaxation. These trajectories are also used in section 3.1 for comparison. The reason to select this excitation frequency $\left(2500 \mathrm{~cm}^{-1}\right)$ is from the expectation that the excitation in the lower energy (red) edge of the vibrational transition will induce more PTs. ${ }^{1,2}$

The initial configurations were sampled randomly in the equilibrium MD simulation with intervals of at least 1 ps to obtain mutually independent ones. In the course of the nonequilibrium MD simulations, the kinetic temperature has increased approximately by $22 \mathrm{~K}$.

The averaged nonequilibrium angles $(\theta)$, distances $(R)$, and numbers of $\mathrm{H}$-bonds $(n)$ are shown in Figure $7 \mathrm{a}, \mathrm{b}$, and $\mathrm{c}$, respectively. We will partition the first hydration shell water molecules into two subsets as shown in Figure 3: $\mathrm{w}^{\mathrm{a}}$ donates the one hydrogen bond (H-bond) to $\mathrm{w}^{*}$, and $\mathrm{w}^{\mathrm{b}}$ accepts $\mathrm{H}$-bond from $\mathrm{w}^{*}$. $\theta_{\mathrm{abc}}$ denotes the angle among $\mathrm{a}, \mathrm{b}$ (center), and $\mathrm{c}$ atoms. If "a" is a hydrogen atom in water, the bisector between the two hydrogen atoms in the molecule was chosen. Likewise, distance $R_{\mathrm{ab}}$, and number of $\mathrm{H}$-bonds $n_{\mathrm{ab}}$ are defined. Because $\theta_{\mathrm{O}^{*} \mathrm{O}^{\mathrm{a}} \mathrm{H}^{\mathrm{a}}}$ is sometimes close to $30^{\circ}$, which should be sampled in section 3.1 , we have adopted the following geometric definition for H-bond: $R_{\mathrm{ab}}<4 \AA$ and $\theta_{\mathrm{abc}}<50^{\circ}$.

According to Omta et al. ${ }^{18,19}$ and Laage et al., ${ }^{20}$ the main contribution of the relaxation process is the first solvation shell of PT complex as shown in Figure 3. The RISM-SCF calculation has shown, in addition, that the distinction of HF from other hydrogen halides is the hydration structure of $\mathrm{F}^{21}$ Hence, in this section, we focus on the dynamics up to the first solvation shell around the $\mathrm{HF}$ and $\mathrm{H}_{2} \mathrm{O}$ molecules shown in Figure 3.

\subsection{Solvent Reorganization}

We begin by examining the nonequilibrium solvation processes and the PT after the impulsive excitation of the HF stretching mode. The results are displayed in Figure 7. Figure 7 (a) shows that $\theta_{\mathrm{O}^{*} \mathrm{O}^{\mathrm{b}} \mathrm{H}^{\mathrm{b}}}$ slightly increases by about $4^{\circ}$ for the reactive trajectories and decreases by about $10^{\circ}$ for the quenched trajectories. The former means that although the dipole moment direction of $\mathrm{w}^{*}$ (the red arrow in Figure 3 ) drastically changes when protonated, $\mathrm{w}^{\mathrm{b}}$ only slightly align to this direction subject to the constraint from the H-bond network. This can also be understood 

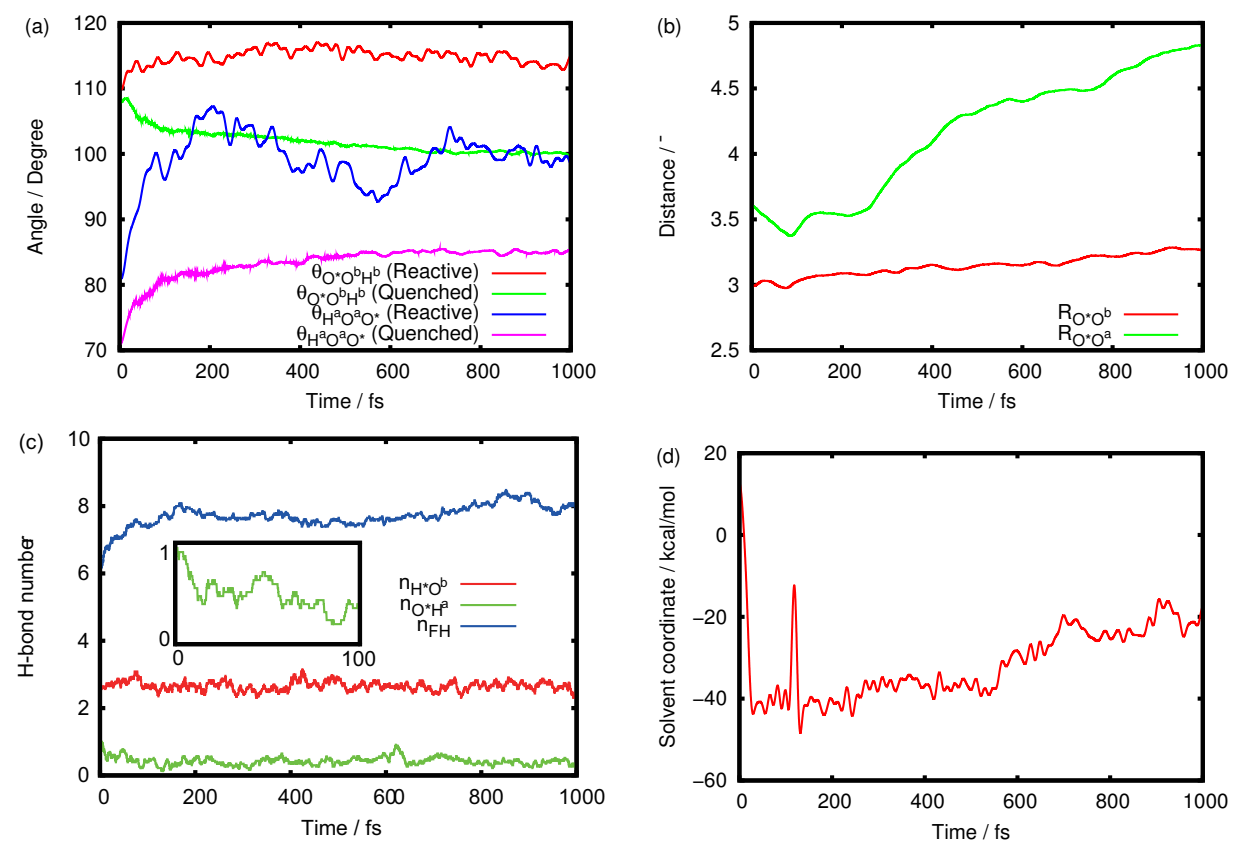

Figure 7: Time evolution for the solvation process after HF dissociation by increasing the velocity of the HF stretching mode in the direction that the HF distance extends by the amount that the kinetic energy increases by $10.1 \mathrm{kcal} / \mathrm{mol}$ corresponding to $2500 \mathrm{~cm}^{-1}$ to break the HF bond by perforce. Averaged (a) angle $\theta$, (b) distance $R$, (c) number of $\mathrm{H}$-bonds $n$, and (d) solvent coordinate. "Reactive" in the legend of (a) means the average over the trajectories in which PT happens. On the other hand, 'Quenched" in the legend of (a) means that the excitation is quenched by vibrational relaxation so that the PT does not happen. The inset in (c) shows the blowup of $n_{\mathrm{O}^{*} \mathrm{H}^{\mathrm{a}}}$ in short time. These quantities and labels are defined in text and Figure 3. 
by the fact that the equilibrium angle $\theta_{\mathrm{O}^{*} \mathrm{O}^{\mathrm{b}} \mathrm{H}^{\mathrm{b}}}$ in an excess proton in water is between $130^{\circ}$ and $160^{\circ}$ by the MS-EVB3 model we have employed. ${ }^{7,22}$ When the PT is quenched, the $\mathrm{F}$ and $\mathrm{O}^{*}$ atoms begin to separate from each other, giving rise to rearrangement of the H-bond network

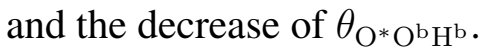

On the other hand, $\theta_{\mathrm{H}^{\mathrm{O}} \mathrm{O}^{\mathrm{O}} \mathrm{O}^{*}}$ gradually increase by approximately $20-25^{\circ}$, indicating that the $\mathrm{w}^{\mathrm{a}}$ actually rotates to break the H-bond to $\mathrm{w}^{*}$ as shown in Figure 3. The decays contain an oscillation mainly due to libration. The quenched trajectory of $\theta_{\mathrm{H}^{\mathrm{O}} \mathrm{O}^{\mathrm{O}} \mathrm{O}^{*}}$ also shows an increase after the excitation, associated with the dissociation of the $\mathrm{FO}^{*} \mathrm{H}$-bond. A notable difference between the reactive and quenched trajectories is seen in their initial values at $t=0$ : the value for the reactive trajectory $\left(\sim 80^{\circ}\right)$ is about $10^{\circ}$ larger than that for the quenched one. This indicates that only the "presolvated" structures closer to the product state, with the more rotated $\mathrm{w}^{\mathrm{a}}$, can undergo PT by excitation. In other words, the determining mechanism is the rotation of the water molecule neighboring to the proton acceptor, which supports the "presolvation mechanism" ${ }^{4,23-25}$ for the PT in a dilute hydrofluoric acid solution.

Next, in Figure $7 \mathrm{~b}, R_{\mathrm{O}^{*} \mathrm{O}^{\mathrm{b}}}$ gradually increases with only a small amount due to diffusion of $\mathrm{w}^{\mathrm{b}}$ molecules because $\mathrm{H}^{*} \mathrm{O}^{\mathrm{b}} \mathrm{H}$-bond becomes relatively strong upon protonation. Conversely, $R_{\mathrm{O}^{*} \mathrm{O}^{\mathrm{a}}}$ begins to increase notably after $t \sim 300 \mathrm{fs}$, when the rotational relaxation of $\theta_{\mathrm{H}^{\mathrm{O}} \mathrm{O}^{\mathrm{a}} \mathrm{O}^{*}}$ has almost completed. This indicates the following mechanism of nonequilibrium solvation: First, $\mathrm{w}^{\mathrm{a}}$ rotates (breaks and reforms $\mathrm{H}$-bonds) in the $\mathrm{H}$-bond network of water, and then $\mathrm{w}^{\mathrm{a}}$ and $\mathrm{w}^{*}$ begins to translate. This picture agrees with the previous results for the equilibrated hydration structure at the TS, in which the angle is more similar to the product state than the distance. ${ }^{4}$

Additionally, in Figure $7 \mathrm{c}$, the number of $\mathrm{H}$-bonds $n_{\mathrm{H}^{*} \mathrm{O}^{\mathrm{b}}}$ thermally fluctuates for the time observed, which shows the stability of this H-bond irrespective of whether before or after PT, whereas upon PT $n_{\mathrm{FH}}$ gradually increases on a similar time scale as for the angles in Figure 7 (a). In the meantime, $n_{\mathrm{O}^{*} \mathrm{H}^{\mathrm{a}}}$ displays a rapid initial decrease by $\sim 0.5$ because of the $\mathrm{H}$-bond breaking due to the net +1 charge of proton-accepting hydronium cation. Finally, in Figure 7 (d), the solvent coordinate shows a sharp drop upon PT mainly due to libration and gradually increases to that in a reactant state chiefly by translation. The sharp rise at $\sim 100 \mathrm{fs}$ is due to a recrossing event of one trajectory. These results are again consistent with the presolvation mechanism discussed above.

The rotation of $\mathrm{w}^{\mathrm{a}}$ needs the $\mathrm{H}$-bond reorganizations outside its first solvation shell. Additionally, the reorganizations of the H-bond topology is also necessary around the $\mathrm{O}^{\mathrm{F}}, \mathrm{O}^{\mathrm{a}}$, and $\mathrm{O}^{\mathrm{b}}$ atoms. ${ }^{7}$ These H-bond reorganizations occur outside the first solvation shell on a picosecond time scale in the HF dissociation in water. This kind of reorganization is expected to be general in proton transfer/transport in water. For example, the recent terahertz time domain spectroscopy in $\mathrm{HCl}$ solution ${ }^{26}$ and the $\mathrm{MD}$ simulation of the $\mathrm{H}$-bond reorientation in anionic hydration shells support this picture. ${ }^{20}$

Moreover, these nonequilibrium MD results underpin the reaction path analysis in section 2.2, which has been derived from the equilibrium $\mathrm{MD}$, in the sense that the librational motion (also FO H-bond vibration and hindered translation) of water is mainly coupled to the PT in the intermolecular interactions.

\subsection{Vibrational Relaxation}

We now turn to the results for the vibrational relaxation of HF and the nearest-neighbor water molecule. Figure 8a shows that the averaged HF distance relaxes on the time scale of $\sim 11 \mathrm{fs}$ (from exponential fitting), which is much faster than the solvent reorganization time in Figure 

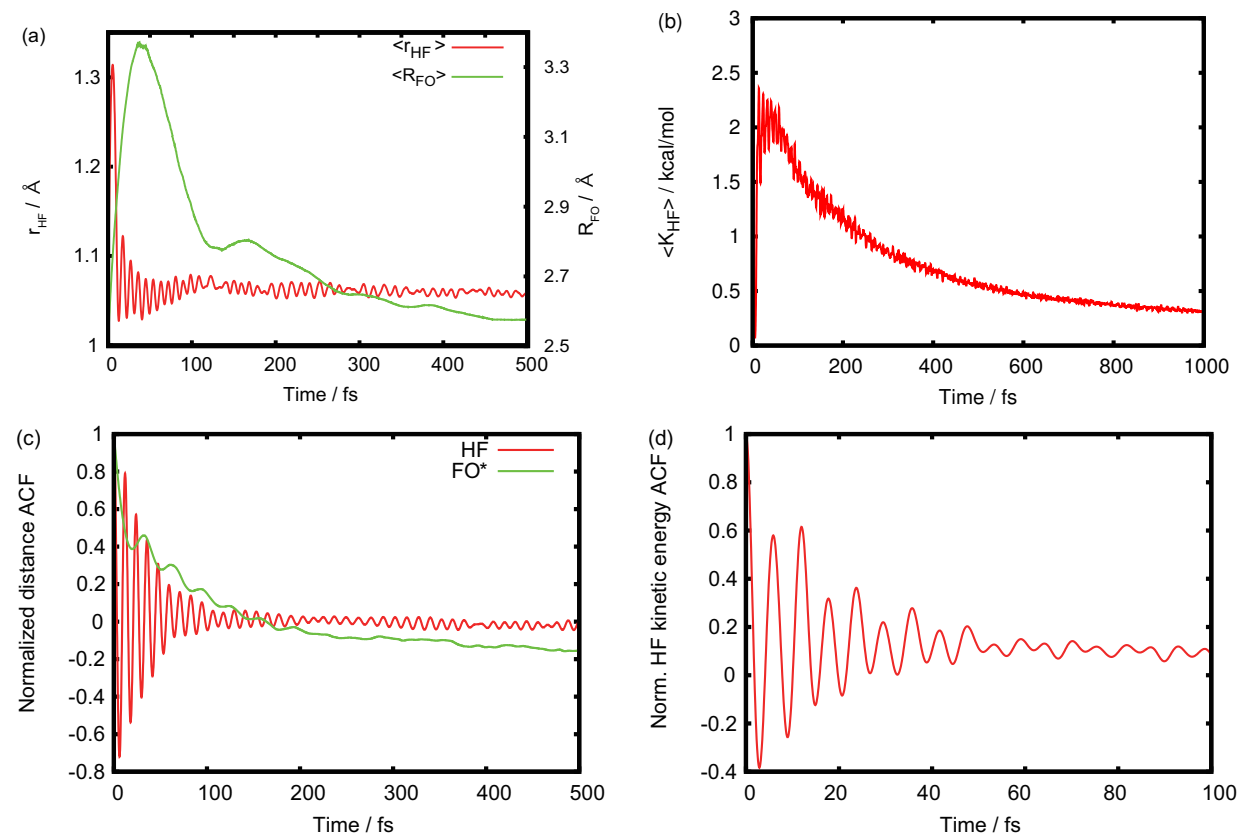

Figure 8: Time evolution of the vibrational relaxation after an IR pulse excitation and the equilibrium autocorrelation functions (ACF). (a) Averaged HF and FO* distances. (b) Averaged HF kinetic energy $K_{\mathrm{HF}}$. (c) Normalized ACF of HF and FO* distances. (d) Normalized ACF of HF stretching energy. Normalizations were carried out by subtracting the equilibrium value and dividing by the initial value.

6. This fast time scale is about one cycle of the HF stretch and can be explained as follows: The excitation energy is so high that, in a half cycle of the $\mathrm{HF}$ stretch oscillation, the $\mathrm{H}^{\mathrm{F}}$ atom pushes the $\mathrm{O}^{*}$ atom to enlarge the FO distance as large as $2.9 \AA$. Then the proton potential around the bottom of the well becomes more harmonic with a larger curvature than in the first oscillation. Consequently, the HF distance becomes unable to stretch further than the first oscillation. After the first rapid oscillation, the HF distance relaxes more slowly on a similar time scale as the solvent reorganization time. For instance, when the FO distance in the nonequilibrium MD simulation is constrained by the RATTLE algorithm, ${ }^{27}$ the first rapid decay (as well as the long HF stretch itself) is not observed.

On the other hand, Figure $8 \mathrm{~b}$ shows that the averaged kinetic energy of HF stretch, $\left\langle K_{\mathrm{HF}}\right\rangle=$ $\mu \dot{r}_{\mathrm{HF}}^{2} / 2$, where $\mu$ is the reduced mass of the diatomic, relaxes on the time scale of $300 \mathrm{fs}$, which is slower than the solvent reorganization time. For the IR-induced PT to occur, the vibrational lifetime should be longer than the solvent reorganization time. Our results indicate that the HF vibrational relaxation and the solvent reorganization compete with each other. In addition, the FO H-bond vibration should affect the vibrational deactivation rate because the ground state potential barrier has been circumvented in effect by the vibrational excitation. In this regard, the averaged FO distance in Figure 8a shows an approximate lifetime of $183 \mathrm{fs}$. Since the FO distance damps slower than the averaged HF distance but faster than the averaged kinetic energy relaxation, the FO H-bond vibration affects the dynamics of PT and vibrational relaxation. For example, when the FO distance is constrained by the RATTLE algorithm ${ }^{27}$ in the nonequilibrium MD simulation, the number of reactive trajectories decreased by a factor of 10 and the energy relaxation time increases to $862 \mathrm{fs}$.

By contrast, the equilibrium ACF of the HF distance in Figure 8c shows an underdamped 
oscillation with a decay time of $\sim 40$ fs of the envelope. On the other hand, the equilibrium ACF of the FO distance display an overdamped decay with small oscillation with the exponential decay time scale of $80 \mathrm{fs}$. This is slightly faster than the corresponding nonequilibrium dynamics in Figure 8a, but is slower than that of the HF distance. This discrepancy between the equilibrium ACFs and the nonequilibrium response arises from the nonlinear dynamics due to the large anharmonicity in the HF stretching PES involved after the excitation. The results also indicate that the anharmonicity along the HF stretch is stronger than that along the FO H-bond.

In summary, the present results indicate that the increase of the vibrational relaxation rate along the excitation energy ${ }^{28,29}$ can be considered as a consequence of the increased anharmonicity. As seen in Figure 2, there is no bath mode of water molecules that matches with the HF stretching frequency. After the excitation, however, the HF stretch starts to be able to couple with the low frequency solvent modes, such as the water bending, which increases the rate of energy relaxation. For instance, the large anharmonic shift of HF stretching frequency is seen in the experiment of HF in pyridine ${ }^{30}$ and in the CPMD simulation. ${ }^{9}$ Moreover, the equilibrium ACF of kinetic energy in Figure 8d decays much faster than the nonequilibrium response in Figure 8b, which underpins the above picture.

\section{Conclusion}

In the present work, we have performed the MS-EVB MD simulations of a dilute hydrofluoric acid solution at an ambient temperature to study the molecular mechanisms in both the vibrational relaxation and the PT induced by the IR excitation.

The investigation by the GLE formalism using the equilibrium simulation shows that the main components of the solvent reorganizations in the PT are the water libration and hindered translation. In particular, the coupling of the water libration to the solvent reorganization is stronger. The nonequilibrium simulation revealed the vibrational relaxation and the solvent reorganization relax on a similar time scale, and competes with each other in the PT. This indicates that the vibrational relaxation can obstruct the IR-induced PT. The rate-limiting step is the rotation of a neighboring water molecule, and the "presolvation mechanism" holds for the PT in a dilute hydrofluoric acid solution. The dynamics of the FO distance was found to accelerate both the PT and the vibrational relaxation. Near the TS of the PT, the vibrational relaxation is notably accelerated due to the increased anharmonicity especially along the HF distance and the resulting enhancement of the nonlinear couplings to the low frequency solvent modes.

\section{Acknowledgment}

T.J. was supported by the Research Fellowship of the Japan Society for the Promotion of Science and the Grants in Aid for Scientific Research (KAKENHI) from the Ministry of Education. K.A. acknowledges support from KAKENHI Nos. 20108017 (“ $\pi$-space”) and 22550012. 


\section{Appendix}

\subsection{Reaction Path Analysis Based on the Generalized Langevin Formal- ism}

In this Appendix, we briefly summarize the reaction path model utilized in section 2.2. ${ }^{13-15}$ Here, we project a large number of the solvent degrees of freedom onto an appropriately defined solvent coordinate $s$ and its perpendicular ones. Because the free energy curves computed in ref 5 are almost parabolic along the diabatic energy gap coordinate and the linear response seems to hold, an appropriate model would be the harmonic one, whose potential energies are thus modeled by

$$
U_{I}(\mathbf{x})=\sum_{i} \frac{\omega_{i}^{2}}{2} x_{i}^{2}+\sum_{i} g_{i}^{I} x_{i}+U_{I}(\mathbf{0}),
$$

where $I=1,2$, corresponding to the reactant and product states, and $x_{i}, \omega_{i}$, and $g_{i}$ are the mass-scaled coordinate, the frequency, and the coupling strength for the $i$ th mode. If we choose the TS point to be the minimum energy point on the crossing surface, $U_{1}(\mathbf{x})=U_{2}(\mathbf{x})$, the directions of the reaction coordinates on the surfaces $U_{1}(\mathbf{x})$ and $U_{2}(\mathbf{x})$ coincide with each other and are normal to the crossing surface at the minimum energy point. Therefore, the reaction coordinate and the normal coordinate can be utilized to describe the dynamics near the TS. Given the potential functions, the minimum energy crossing point $\mathrm{x}^{\ddagger}$ is given by

$$
x_{i}^{\ddagger}=\omega_{i}^{-2}\left\{\sum_{j} \omega_{j}^{-2}\left(g_{i}^{1} g_{j}^{2}-g_{i}^{2} g_{j}^{1}\right) \Delta g_{j}+\Delta g_{i} s(\mathbf{0})\right\} / \sum_{j} \omega_{j}^{-2} \Delta g_{j}^{2}
$$

with $\Delta g_{j}=g_{j}^{2}-g_{j}^{1}$ and the solvent coordinate is defined here by $s(\mathbf{x})=U_{2}(\mathbf{x})-U_{1}(\mathbf{x})$. Now we write the direction vector of reaction coordinate ${ }^{t} \tilde{\mathbf{s}}_{i}=\left(\tilde{s}_{1}, \tilde{s}_{2}, \cdots\right)$ as

$$
\tilde{s}_{i}=\frac{d x_{i}}{d \tilde{s}}=\frac{\Delta g_{i}}{\left[\sum_{j}\left(\Delta g_{j}\right)^{2}\right]^{1 / 2}}
$$

If we take the coordinate $\tilde{s}$ as a linear line in the direction of $\tilde{\mathbf{s}}, \tilde{s}={ }^{t} \tilde{\mathbf{s}} \cdot \tilde{\mathbf{x}}={ }^{t} \tilde{\mathbf{s}} \cdot\left(\mathbf{x}-\mathbf{x}^{\ddagger}\right)$, where $\tilde{\mathbf{x}}$ is the displacement measured from the minimum energy crossing point, the solvent coordinate $s$ is proportional to $\tilde{s}, s=\mu^{-1 / 2} \tilde{s}$, where $\mu$ is the "effective mass" expressed by the difference of the coupling strength $\Delta g_{k}$

$$
\mu^{-1 / 2}=\left[\sum_{k}\left(\Delta g_{k}\right)^{2}\right]^{1 / 2}
$$

Using the projection operator $\hat{\mathbf{P}}$ with the element $\hat{P}_{i j}=\Delta g_{i} \Delta g_{j} /\left[\sum_{k}\left(\Delta g_{k}\right)^{2}\right]$, and the usual matrix partitioning technique with $\hat{\mathbf{Q}}=\mathbf{I}-\hat{\mathbf{P}}$, we can derive the generalized Langevin equation (GLE) for the solvent coordinate

$$
\ddot{s}+\Omega^{2} s+\mu^{-1 / 2} \bar{g}+\int_{0}^{t} \zeta(\tau) \dot{s}(t-\tau) d \tau+\zeta(t) s(0)=\mu^{-1 / 2} R(t),
$$

in which $\bar{g}=\sum_{k}\left(g_{k}^{I}+\omega_{k}^{2} x_{k}^{\ddagger}\right) \tilde{s}_{k}$. The friction kernel $\zeta(t)$ is given formally as

$$
\zeta(t)=\tilde{\omega}_{P Q}^{2} \tilde{\omega}_{Q Q}^{-2} \cos \left(\tilde{\omega}_{Q Q} t\right) \tilde{\omega}_{Q P}^{2}
$$


and is related to the random force $R(t)$ by the second fluctuation-dissipation theorem $\langle R(0) R(t)\rangle=$ $\zeta(t) k_{\mathrm{B}} T$. The effective frequency $\Omega$ for the harmonic free energy curve along the solvent coordinate is given by

$$
\Omega^{2}=\bar{\omega}^{2}-\zeta(0)
$$

in which we have defined the average frequency

$$
\bar{\omega}^{2}=\sum_{k} \omega_{k}^{2} \tilde{s}_{k}^{2}
$$

Therefore, $\Omega$ contains the frequency shift from $\bar{\omega}$ due to the coupling to the bath. The elements of column vector $\tilde{\omega}_{Q P}^{2}$ and matrix $\tilde{\omega}_{Q Q}^{2}$ are given by $\tilde{\omega}_{Q P, i}^{2}=\left(\omega_{i}^{2}-\bar{\omega}^{2}\right) \tilde{s}_{i}$, and $\tilde{\omega}_{Q Q, i j}^{2}=\omega_{i}^{2} \delta_{i j}+$ $\left(\bar{\omega}^{2}-\omega_{i}^{2}-\omega_{j}^{2}\right) \tilde{s}_{i} \tilde{s}_{j}$. The raw vector $\tilde{\omega}_{P Q}^{2}$ is the transpose of $\tilde{\omega}_{Q P}^{2}$.

The present MD calculations provide the quantities in the GLE, eq 6, in the following way. First, the components of direction vector $\tilde{\mathbf{s}}$ and corresponding frequencies $\omega_{i}$ are computed with the Fourier transform of the velocity autocorrelation function

$$
\langle\dot{s}(0) \dot{s}(t)\rangle=\frac{k_{\mathrm{B}} T}{\mu} \sum_{i} \tilde{s}_{i}^{2} \cos \omega_{i} t .
$$

From eq 10, $\mu$ and $\tilde{s}_{i}^{2}$ are calculated using the equilibrium MD trajectories (usually in the reactant state), and then the key quantities, such as $\zeta(t)$ and $\Omega$ are constructed from eqs 7 and 8 , respectively.

\section{References}

[1] Hammes-Schiffer, S.; Tully, J. C. J. Phys. Chem. 1995, 99, 5793.

[2] Ando, K.; Staib, A.; Hynes, J. T. Femtochemistry: Ultrafast Chemical and Physical Processes in Molecular Systems; World Scientific: Singapore, 1996.

[3] Hanna, G.; Geva, E. J. Phys. Chem. B 2008, 112, 4048-4058.

[4] Ando, K.; Hynes, J. T. J. Phys. Chem. A 1999, 103, 10398.

[5] Strasfeld, D. B.; Shim, S.-H.; Zanni, M. T. Adv. Chem. Phys. 2009, 141, 1-28.

[6] Engel, V.; Meier, C.; Tannor, D. J. Adv. Chem. Phys. 2009, 141, 29-101.

[7] Joutsuka, T.; Ando, K. J. Phys. Chem. A 2010, DOI: 10.1021/jp108147e.

[8] Humphrey, W.; Dalke, A.; Schulten, K. J. Mol. Graphics. 1996, 14, 33.

[9] Iftimie, R.; Thomas, V.; Plessis, S.; Marchand, P.; Ayotte, P. J. Am. Chem. Soc. 2008, 130, 5901-5907.

[10] Iftimie, R.; Tuckerman, M. E. Angew. Chem., Int. Ed. 2006, 45, 1144-1147.

[11] Laasonen, K.; Klein, M. L. Mol. Phys. 1996, 88, 135-142.

[12] Laage, D.; Demirdjian, H.; Hynes, J. T. Chem. Phys. Lett. 2005, 405, 453-458. 
[13] Ando, K.; Kato, S. J. Chem. Phys. 1991, 95, 5966-5982.

[14] Hayashi, S.; Ando, K.; Kato, S. J. Phys. Chem. 1995, 99, 955-964.

[15] Ando, K. J. Chem. Phys. 1994, 101, 2850-2862.

[16] Staib, A.; Borgis, D.; Hynes, J. T. J. Chem. Phys. 1995, 102, 2487-2505.

[17] Maroncelli, M. J. Mol. Liq. 1993, 57, 1-37.

[18] Omta, A. W.; Cropman, M. F.; Woutersen, S.; Bakker, H. J. Science 2003, 301, 347.

[19] Bakker, H. J. Chem. Rev. 2008, 108, 1456-1473.

[20] Laage, D.; Hynes, J. T. Proc. Nat. Am. Soc. 2007, 104, 11167-11172.

[21] Sato, H.; Hirata, F. J. Am. Chem. Soc. 1999, 121, 3460-3467.

[22] Wu, Y.; Chen, H.; Wang, F.; Paesani, F.; Voth, G. A. J. Phys. Chem. B 2008, 112, 467-482.

[23] Marx, D.; Chandra, A.; Tuckerman, M. E. Chem. Rev. 2010, 110, 2174-2216.

[24] Ayotte, P.; Plessis, S.; Marchand, P. Phys. Chem. Chem. Phys. 2008, 10, 4785-4792.

[25] Ando, K.; Hynes, J. T. J. Phys. Chem. B 1997, 101, 10464-10478.

[26] Tielrooij, K. J.; Timmer, R. L. A.; Bakker, H. J.; Bonn, M. Phys. Rev. Lett. 2009, 102, 198303-198306.

[27] Anderson, H. C. J. Comput. Phys. 1983, 52, 24-34.

[28] Roberts, S. T.; Petersen, P. B.; Ramasecha, K.; Tokmakoff, A.; Ufimtsev, I. S.; Martinez, T. J. Proc. Nat. Am. Soc. U.S.A. 2009, 111, 15154-15159.

[29] Bader, J. S.; Berne, B. J.; Pollak, E.; Hänggi, P. J. Chem. Phys. 1996, 104, 1111-1119.

[30] van den Broek, M. A. F. H.; Kropman, M. F.; Bakker, H. J. Chem. Phys. Lett. 2002, 357, 8-14. 


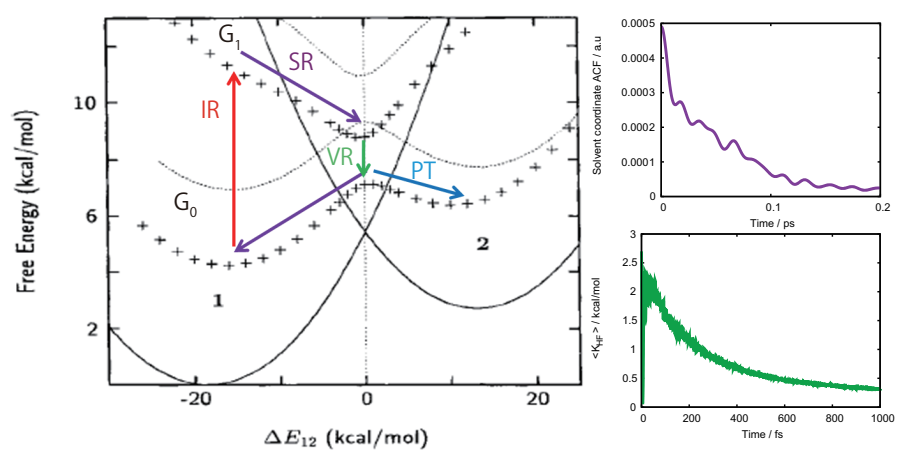

Table of contents graphics 